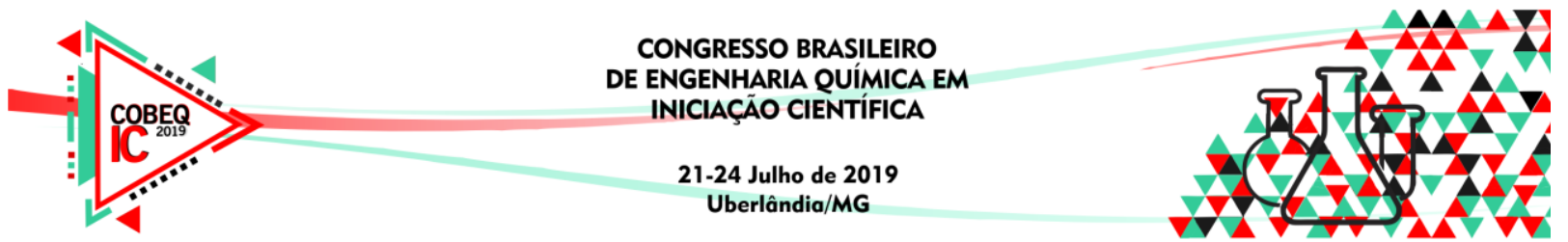

\title{
SECAGEM DA MISTURA FERTILIZANTE-BIOMASSA PARA PRODUÇÃO DE FERTILIZANTES DE LIBERAÇÃO LENTA
}

\author{
T. M. JESUS ${ }^{1}$, G. F. TEIXEIRA ${ }^{1}$, K.G. SANTOS ${ }^{1}$ \\ ${ }^{1}$ Universidade Federal do Triangulo Mineiro, Faculdade de Engenharia Química \\ E-mail para contato: kassia.santos@uftm.edu.br
}

\begin{abstract}
RESUMO - O estudo de novas formas de aproveitamento de fertilizantes tem ganhado cada vez mais destaque, já que ainda há uma grande perda dos nutrientes para o solo, por processos como lixiviação e volatilização. Diante disto, este trabalho estudou a impregnação de fertilizante NPK em biomassa, a fins de retardar sua liberação. Como forma de aglomeração, as amostras com diferentes concentrações de fertilizante e malte passaram pelo processo de secagem em um secador de infravermelho. Foi avaliado o efeito da concentração de fertilizante, da temperatura e do tempo de embebimento do fertilizante na biomassa, sobre a cinética de secagem, observando-se que a temperatura é a variável mais significativa. A cinética deste processo foi melhor representada pelo modelo de Page. A partir de medidas de condutividade foi avaliada a liberação das amostras aglomeradas, sendo que as amostras com 1,5 g fertilizante/g biomassa, secas à $60^{\circ} \mathrm{C}$ apresentaram a liberação de nutrientes mais lenta.
\end{abstract}

\section{INTRODUÇÃO}

Nos dias atuais, o sistema agrícola é essencial para a produção de alimentos e bioenergia. Sendo assim, o uso dos fertilizantes possui extrema importância, pois são utilizados na reposição de nutrientes para as plantas, eles fornecem um ou mais nutrientes que são necessários para que haja um desenvolvimento satisfatório das plantas (SILVA; LOPES, 2011).

Alguns nutrientes essenciais muitas vezes não são muito aproveitados pelas plantas devido aos processos de imobilização, desnitrificação, volatilização e lixiviação que acontecem no solo (ALMEIDA; SANCHES, 2012). Diante disso, outras alternativas vêm sendo buscadas para que haja um maior aproveitamento de nutrientes pelo solo, ou seja, uma eficiência maior dos fertilizantes. Uma alternativa encontrada é a utilização de fertilizantes de liberação lenta. Este tipo de fertilizante é responsável por aumentar o tempo em que o nutriente ficará disponível ao solo. Isto ocorre quando os fertilizantes solúveis são revestidos ou aglomerados (AZEEM et al., 2014).

Este trabalho tem como objetivo, desenvolver um fertilizante imobilizado em biomassa, com a finalidade de retardar sua liberação. Assim, estudou-se o efeito da razão mássica de fertilizante para biomassa, da temperatura e do tempo de embebimento do fertilizante na biomassa, sobre a cinética da secagem da mistura (parâmetros cinéticos do melhor modelo de secagem e sobre o tempo em que $90 \%$ da umidade foi removida). Posteriormente, foi realizada a analise de liberação do fertilizante, para selecionar a melhor condição de secagem e composição do fertilizante. 


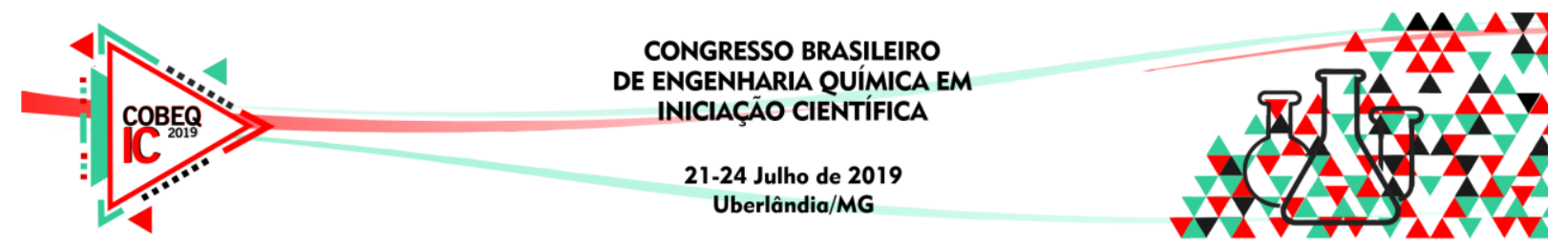

\section{MATERIAIS E MÉTODOS}

\subsection{Caracterização da biomassa}

Neste trabalho, a biomassa utilizada foi o resíduo de malte, obtido em uma cervejaria de Uberaba. A densidade aparente das partículas foi obtida por picnometria utilizando éter de petróleo. A análise morfológica das partículas foi feita a partir da análise de fotos por meio do software ImageJ, através das quais obteve-se os fatores de forma das partículas de malte, a saber, a circularidade, o alongamento e a esfericidade, representados pelas Equações 1 a 3, respectivamente (CREMASCO, 2012).

$$
\begin{aligned}
& C=\frac{P^{2}}{4 \pi S p} \\
& A l=\frac{b}{a} \\
& \phi=\frac{A_{p} 4 \pi}{P^{2}}
\end{aligned}
$$

sendo que, e $S p$ a área superficial da partícula e $P$ é o perímetro; $b$ é a maior dimensão da partícula e $a$, a menor e $A_{p}$ é a área projeta.

A distribuição granulométrica das partículas de malte foi obtida por peneiramento. Com os dados obtidos calculou-se o diâmetro médio de Sauter, através da Equação 4, e foram ajustados os modelos de distribuição granulométrica GGS, RRB e Sigmóide, representados pelas Equações de 5 a 7, respectivamente (CREMASCO, 2012).

$$
D_{\text {sauter }}=\frac{1}{\sum_{n=1}^{n} \frac{\Delta x_{n}}{\overline{D_{n}}}}
$$

em que $\bar{D}_{n}$ é o diâmetro médio entre duas peneiras e $\Delta x_{n}$ é a fração mássica retida em cada peneira.

$$
\begin{aligned}
& X=\left(\frac{D}{k}\right)^{m} \\
& X=1-e^{-\left(\frac{D}{D^{\prime}}\right)^{n}} \\
& X=\left[1+\frac{D_{50}}{D}\right]^{-p}
\end{aligned}
$$

\subsection{Planejamento Experimental}

A fim de obter o efeito das variáveis razão mássica de fertilizante/biomassa (C), temperatura de secagem (T) e tempo de embebimento da amostra (t), sobre a cinética de secagem, foram realizados experimentos segundo um planejamento composto central, totalizando 20 experimentos. O efeito das variáveis sobre as respostas foram avaliados empregando a metodologia de superfície de respostas, com nível de confiança de $10 \%$. 


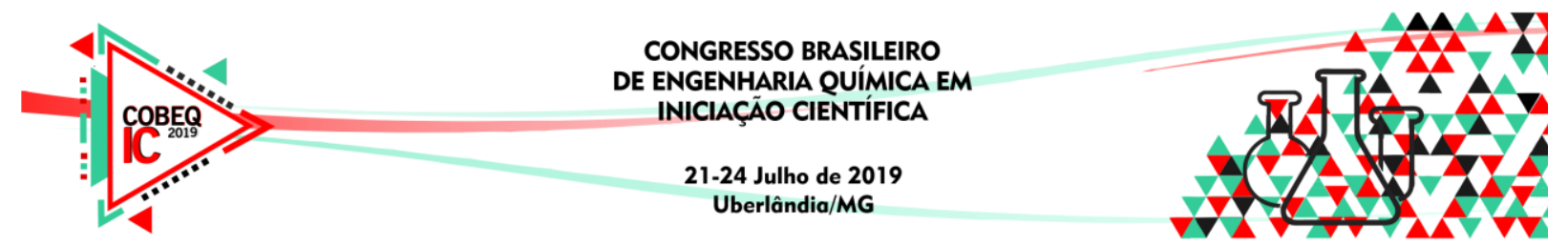

\subsection{Cinética de secagem}

As amostras foram preparadas nas concentrações indicadas pelo planejamento e impregnadas, seguindo o tempo de embebimento de cada experimento. Em seguida a amostra foi submetida ao teste de secagem. Foi utilizado um analisador de umidade infravermelho para a obtenção da massa da mistura e avaliação da cinética de secagem das amostras de fertilizante com malte. Os valores do percentual de umidade da amostra que é perdida com o tempo são fornecidos pelo analisador e foram anotados em intervalos de tempo de 1 minuto até a estabilização. Com o percentual fornecido, foi possível calcular pelas Equações 8 a 10, a umidade em base seca $(X b s)$, a razão de umidade $(R U)$ e a taxa de secagem $(N)$ respectivamente; em que $X b s i$ é a umidade em base seca no instante $i, m a$ é a massa de água, $m s$ a massa seca do material, $X e$ a umidade de equilíbrio dinâmica e $X b s_{0}$ a umidade inicial; $R U$ é a razão de umidade; $\mathrm{t}$ o tempo de secagem; $\mathrm{k}, \mathrm{k} 0$ e $\mathrm{k} 1$ as constantes de secagem; $\mathrm{a}, \mathrm{b}, \mathrm{c}$ e $\mathrm{n}$ os coeficientes dos modelos.

$$
\begin{aligned}
& X b s i=\frac{m a}{m s} \\
& R U=\frac{(X b s i-X e)}{\left(X b s_{0}-X e\right)} \\
& N=\frac{\Delta R U}{\Delta t}
\end{aligned}
$$

Vários modelos cinéticos de secagem encontrados na literatura (Tabela 1) foram utilizados para o ajuste desses dados (RESENDE; FERREIRA; ALMEIDA, 2010).

Tabela 1- Modelos de cinética de secagem

\begin{tabular}{lcc}
\hline \multicolumn{1}{c}{ Nome } & Modelo & Equação \\
\hline Wang e Singh & $R U=1+a t+b t^{2}$ & $(11)$ \\
Verna & $R U=a \exp (-k t)+(1-a) \exp (-b t)$ & $(12)$ \\
Page & $R U=\exp \left(-k t^{n}\right)$ & $(13)$ \\
Newton & $R U=\exp (-k t)$ & $(14)$ \\
Midilli & $R U=a \exp \left(-k t^{n}\right)+b t$ & $(15)$ \\
Logaritmo & $R U=a \exp (-k t)+c$ & $(16)$ \\
Henderson e Pabis & $R U=a \exp (-k t)$ & $(17)$ \\
Henderson e Pabis modificado & $R U=a \exp \left(-k_{0} t\right)+b \exp \left(k_{1} t\right)+c \exp \left(k_{2} t\right)$ & $(18)$ \\
Exponencial de dois termos & $R U=a \exp (-k t)+(1-a) \exp (-k a t)$ & $(19)$ \\
Dois termos & $R U=a \exp (-k t)+b \exp (-k t)$ & $(20)$ \\
Aproximação por difusão & $R U=a \exp (-k t)+(1-a) \exp (-k b t)$ & $(21)$ \\
\hline
\end{tabular}

A equação de Arrhenius (Equação 22), pode substituir a constante de secagem; sendo que $A$ é o fator de frequência, $E a$ é a energia de ativação $[\mathrm{kJ} / \mathrm{mol}], R$ é a constante dos gases $(8,13$ $J / K . m o l)$ e $T$ a temperatura $[K]$.

$$
k=A \cdot \exp ^{\frac{-E a}{R T}}
$$

\subsection{Teste de liberação fertilizante in vitro}

Para o teste da liberação do fertilizante consistiu na adição da amostra imobilizada em um sachê à $250 \mathrm{~mL}$ de água, sob agitação. A concentração do fertilizante dissolvido ao longo do tempo foi obtida por meio das medidas online de condutividade, com o software Logger Lite. 


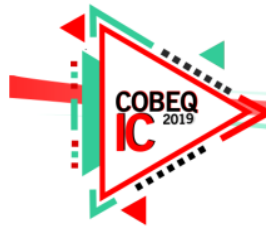

\section{RESULTADOS}

A Tabela 2 apresenta as propriedades da análise morfológica, de tamanho e densidade das partículas. Pode-se observar que o valor do alongamento obtido é maior que 1 , o que significa que a partícula é alongada, com esfericidade de 0,46 e circularidade de 1,36. Através da picnometria das partículas de malte com éter de petróleo, foi definida a densidade aparente de 0,40 g/ $\mathrm{cm}^{3}$ (SILVÉRIO, 2018). Pelo peneiramento, foi possível calcular um diâmetro médio de Sauter de 2,11 mm. O modelo que melhor caracterizou a distribuição de tamanho das partículas foi o modelo Sigmóide.

Tabela 2 - Propriedades e distribuição granulométrica das partículas de biomassa.

\begin{tabular}{|c|c|c|c|c|c|}
\hline \multicolumn{2}{|c|}{ Propriedades da partícula } & \multicolumn{4}{|c|}{ Distribuição Granulométrica } \\
\hline & & Modelo & $\mathrm{R}^{2}$ & & etros \\
\hline Esfericidade & 0,46 & GGS & 0,9592 & $K[\mathrm{~mm}]$ & 4,7657 \\
\hline Alongamento & 2,81 & & & $M$ & 1,9947 \\
\hline Circularidade & 1,36 & RRB & 0,9936 & $D^{\prime}[\mathrm{mm}]$ & 3,5144 \\
\hline Densidade aparente $\left[\mathrm{g} / \mathrm{cm}^{3}\right]$ & 0,40 & & & $N$ & 4,2477 \\
\hline Diâmetro de Sauter [mm] & 2,11 & Sigmóide & 0,9937 & $D_{50}[\mathrm{~mm}]$ & 3,1315 \\
\hline
\end{tabular}

Na Figura 1 estão representadas as razões de umidade variando com o tempo para cada uma das amostras analisadas. Pode-se observar que a razão de umidade decresce mais rápido para as amostras que foram expostas à maiores temperaturas. A amostra 12 que foi analisada à $110^{\circ} \mathrm{C}$ e as amostras $3,4,7$ e 8 à $100^{\circ} \mathrm{C}$ apresentaram menores razões de umidade em menor tempo, o que indica uma maior secagem. Todos os modelos de secagem estudados foram ajustados para todas as amostras, sendo o modelo de Page aquele que melhor ajustou os dados experimentais da cinética de secagem.

Figura 1 - Curva de secagem para amostras de: (a) 1 a 5; (b) 6 a 10; (c) 11 a 15; (d) 16 a 20.
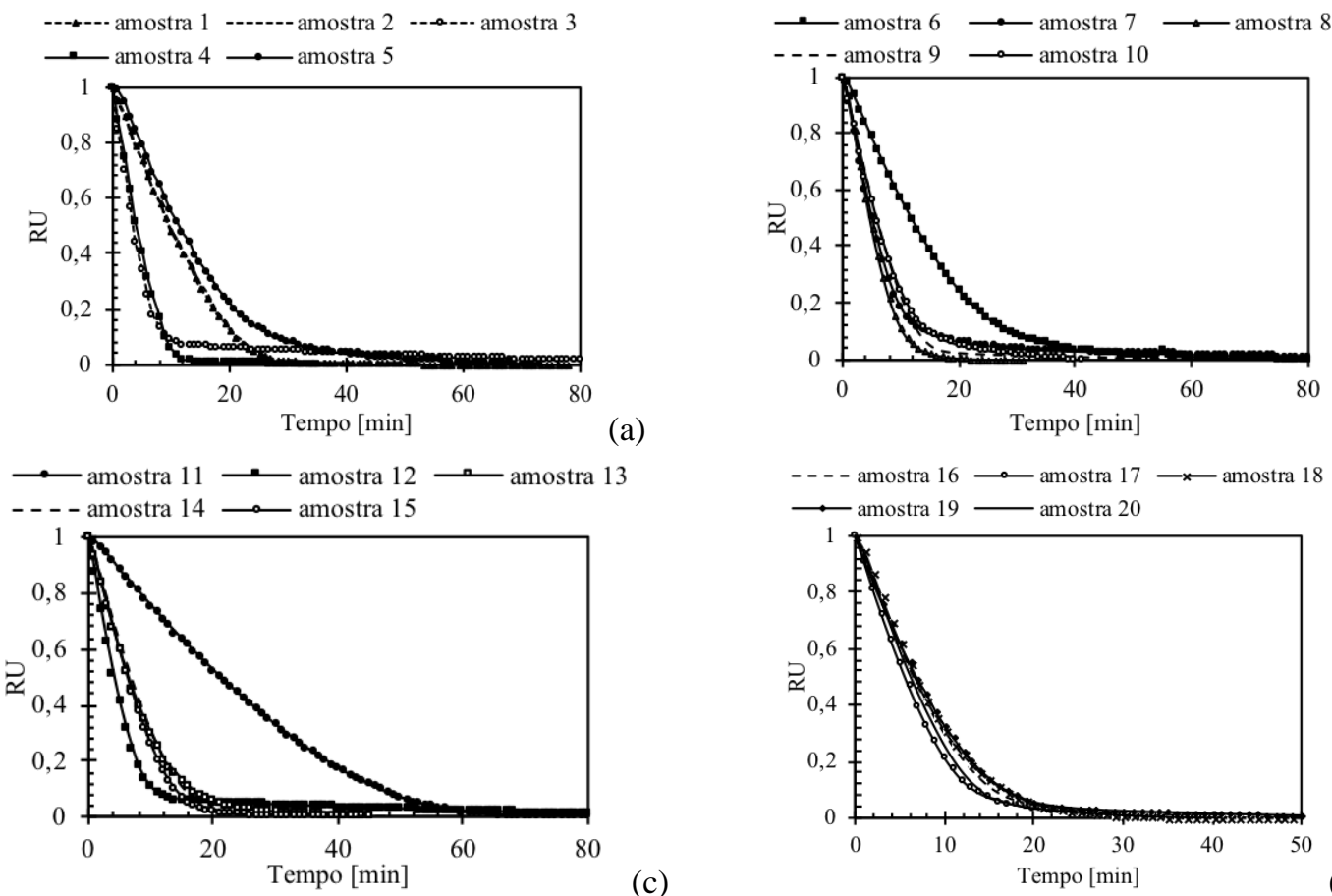


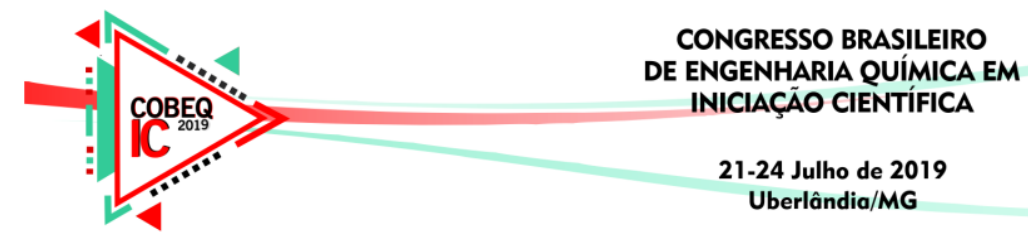

A Tabla 3 apresenta os parâmetros do modelo de Page para cada condição do planejamento experimental, bem como o tempo em que houve a secagem de $90 \%$ da amostra. Foram analisados os efeitos da razão fertilizante/biomassa (X1), temperatura (X2) e tempo de embebimento (X3) sobre os parâmetros k e n do modelo de Page e sobre o t90\%, obtidos pelo software Statistica. As Equações 23 a 25 representam os modelos reduzidos para as respostas k $\left(\mathrm{R}^{2}=0,925\right), \mathrm{n}\left(\mathrm{R}^{2}=0,90\right)$ e t $90 \%\left(\mathrm{R}^{2}=0,947\right)$, respectivamente.

Tabela 3 - Planejamento composto central para a secagem das amostras a serem analisadas.

\begin{tabular}{|c|c|c|c|c|c|}
\hline \multirow[b]{2}{*}{ Amostra } & \multicolumn{3}{|c|}{ Variáveis } & \multicolumn{2}{|c|}{ Respostas } \\
\hline & $\mathrm{C}(\mathrm{g}$ fert/g bio $)$ & $\mathrm{T}\left({ }^{\circ} \mathrm{C}\right)$ & $\mathrm{t}(\min )$ & ts $90 \%(\mathrm{~min})$ & $\begin{array}{l}\text { Parâmetros do } \\
\text { Modelo de Page }\end{array}$ \\
\hline 1 & 0,9 & 60 & 21 & 21 & $\begin{array}{l}\mathrm{n}=1,4328 \\
\mathrm{k}=0,0277\end{array}$ \\
\hline 2 & 0,9 & 60 & 99 & 21,3 & $\begin{array}{l}\mathrm{n}=1,4357 \\
\mathrm{k}=0,0269\end{array}$ \\
\hline 3 & 0,9 & 100 & 21 & 9 & $\begin{array}{l}\mathrm{n}=1,0364 \\
\mathrm{k}=0,1990\end{array}$ \\
\hline 4 & 0,9 & 100 & 99 & 9 & $\begin{array}{l}\mathrm{n}=1,4016 \\
\mathrm{k}=0,0987\end{array}$ \\
\hline 5 & 1,5 & 60 & 21 & 28 & $\begin{array}{l}\mathrm{n}=1,3164 \\
\mathrm{k}=0,0284\end{array}$ \\
\hline 6 & 1,5 & 60 & 99 & 28,5 & $\begin{array}{l}\mathrm{n}=1,2966 \\
\mathrm{k}=0,0286\end{array}$ \\
\hline 7 & 1,5 & 100 & 21 & 14,1 & $\begin{array}{l}\mathrm{n}=1,0965 \\
\mathrm{k}=0,1206\end{array}$ \\
\hline 8 & 1,5 & 100 & 99 & 10,4 & $\begin{array}{l}\mathrm{n}=1,4641 \\
\mathrm{k}=0,0730\end{array}$ \\
\hline 9 & 0,74 & 80 & 60 & 12,9 & $\begin{array}{l}\mathrm{n}=1,3802 \\
\mathrm{k}=0,0624\end{array}$ \\
\hline 10 & 1,66 & 80 & 60 & 15 & $\begin{array}{l}\mathrm{n}=1,2110 \\
\mathrm{k}=0,0841\end{array}$ \\
\hline 11 & 1,2 & 50 & 60 & 46 & $\begin{array}{l}\mathrm{n}=1,4306 \\
\mathrm{k}=0,0091\end{array}$ \\
\hline 12 & 1,2 & 110 & 60 & 10,2 & $\begin{array}{l}\mathrm{n}=1,1319 \\
\mathrm{k}=0,1457\end{array}$ \\
\hline 13 & 1,2 & 80 & 0,54 & 16,5 & $\begin{array}{l}\mathrm{n}=1,2215 \\
\mathrm{k}=0,0733\end{array}$ \\
\hline 14 & 1,2 & 80 & 119 & 15,2 & $\begin{array}{l}\mathrm{n}=1,3903 \\
\mathrm{k}=0,0503\end{array}$ \\
\hline
\end{tabular}

$$
\begin{aligned}
& k=0,061+0,0486 X_{2}+0,0103 X_{2}^{2}-0,0173 X_{3}-0,0132 X_{1} X_{2}-0,0219 X_{2} X_{3} \\
& n=1,332-0,0556 X_{1}-0,0883 X_{2}+0,081 X_{3}+0,049 X_{1} X_{2}+0,099 X_{2} X_{3} \\
& t_{90 \%}=13,1370+1,6371 X_{1}-7,1051 X_{2}+4,4315 X_{2}^{2}-1,2150 X_{1} X_{2}
\end{aligned}
$$

Analisando o efeito das variáveis sobre o tempo gasto para secar $90 \%$ da umidade da amostra, observa-se que a variável X2 (temperatura) é negativa, o que significa que ela influencia inversamente, ou seja, se a temperatura aumenta, o tempo de secagem diminui. Já a variável X1 (razão mássica de fertilizante/biomassa) influencia diretamente o processo, assim o aumento de $\mathrm{C}$ conduz a um maior tempo de secagem. Quanto aos parâmetros do modelo de Page, a velocidade de secagem (k) e sua ordem (n), nota-se que C (X1) e t (X3) influenciam inversamente o $k$ e o $n$. Já com a temperatura, o aumento do $k$ e $n$ ocorre com a temperatura. 


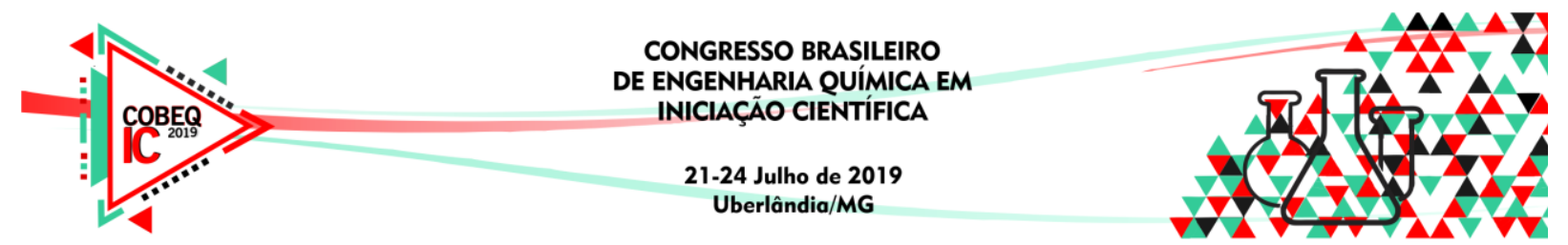

Foram realizadas medidas de condutividade de cada uma das 20 amostras. A Figura 2 mostra a comparação da condutividade com o tempo para o fertilizante puro e as amostras 1 a 8 . Dentre todos os testes, as amostras 5 e 6 (com $1,5 \mathrm{~g}$ fertilizante $/ \mathrm{g}$ biomassa; $\mathrm{T}=60^{\circ} \mathrm{C}$ ) foram as que apresentaram tempo de liberação mais retardado sem alterar a concentração total de fertilizante após a secagem, sendo essa condição recomendada para futuros testes de granulação em outros equipamentos, como secador solar ou tambor rotatório.

Figura 2 - Comparação da variação da condutividade em função do tempo das amostras com o fertilizante: (b) amostras de 1 a 4; (b) de 5 a 8.
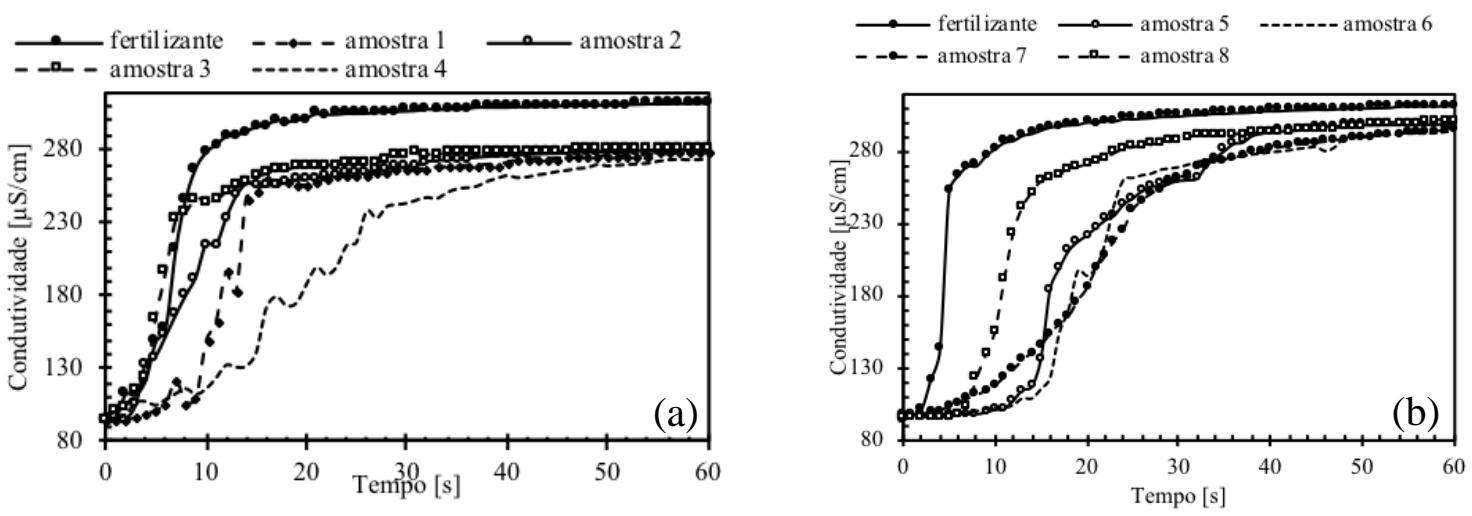

\section{CONCLUSÕES}

Através da secagem no analisador de umidade infravermelho, pode-se concluir que o modelo cinético de secagem que melhor se ajustou à secagem da mistura malte-fertilizante foi o Page. A temperatura é a variável estudada que mais influencia a velocidade de secagem (k), sendo que o tempo de embebimento só se mostrou significativo na forma de interação com a variável temperatura. Ao analisar a liberação do fertilizante em tanque agitado, as amostras 5 e 6 (com 1,5 g fertilizante/g biomassa; $\mathrm{T}=60^{\circ} \mathrm{C}$ ) foram as que apresentaram liberação mais lenta, independente do tempo de embebimento. Assim, indica-se essa condição para futuros testes de granulação em outros equipamentos.

\section{REFERÊNCIAS}

ALMEIDA, R. F.; SANCHES, B. C. Fertilizantes Nitrogenados com liberação lenta e estabilizada na agricultura. Revista Verde de Agroecologia e Desenvolvimento Sustentável, Mossoró, n. ISSN 1981-8203, p. 31-35, dez. 2012.

AZEEM, B.; KUSHAARI, K.; MAN, Z.B.; BASIT, A.; THANH, T. H. Review on materials \& methods to produce controlled release coated urea fertilizer. Journal of Controlled Release, v. 181, 11-21 p, 2014.

CREMASCO, M. A.; Operações unitárias em sistemas particulados e fluidomecânicos. 2. ed. São Paulo: Blucher, 2012. 417 p.

FOUST, A.S., WENZEL, L.A., CLUMP, C.W., MAUS, L., ANDERSEN, L.B., Princípios das Operações unitárias. 2 ed. Rio de Janeiro: LTC. 1982.

MCCABE, W.; SMITH, J.; HARRIOT, P. Unit Operations of Chemical Engineering. 7 ed. McGraw Hill Chemical Engineering Series, 2005.

SILVA, D.; LOPES, A. Princípios básicos para formulação e mistura de fertilizantes. 2011. 53 p. Universidade Federal de Lavras, 2011. 